\title{
Statistical Mechanics of Double sinh-Gordon Kinks
}

\author{
Salman Habib ${ }^{1 *}$, Avinash Khare ${ }^{2}$, and Avadh Saxena ${ }^{3}$ \\ ${ }^{1}$ T-8, Theoretical Division, MS B285, Los Alamos National Laboratory, Los Alamos, New Mexico 87545 \\ ${ }^{2}$ Institute of Physics, Sachivalaya Marg, Bhubaneswar 751 005, India \\ ${ }^{3}$ T-11, Theoretical Division, MS B262, Los Alamos National Laboratory, Los Alamos, New Mexico 87545
}

(August 27, 2018)

\begin{abstract}
We study the classical thermodynamics of the double sinh-Gordon (DSHG) theory in $1+1$ dimensions. This model theory has a double well potential $V(\phi)=(\zeta \cosh 2 \phi-n)^{2}$ when $n>\zeta$, thus allowing for the existence of kinks and antikinks. Though it is nonintegrable, the DSHG model is remarkably amenable to analysis. Below we obtain exact single kink and kink lattice solutions as well as the asymptotic kink-antikink interaction. In the continuum limit, finding the classical partition function is equivalent to solving for the ground state of a Schrödinger-like equation obtained via the transfer integral method. For the DSHG model, this equation turns out to be quasi-exactly solvable. We exploit this property to obtain exact energy eigenvalues and wavefunctions for several temperatures both above and below the symmetry breaking transition temperature (provided $n=1,2, \cdots, 6$ ). The availability of exact results provides an excellent testing ground for large scale Langevin simulations. The probability distribution function (PDF) calculated from Langevin dynamics is found to be in striking agreement with the exact PDF obtained from the ground state wavefunction. This validation points to the utility of a PDF-based computation of thermodynamics utilizing Langevin methods. In addition to the PDF, field-field and field fluctuation correlation functions were computed and also found to be in excellent agreement with the exact results.
\end{abstract}

PACS numbers: 05.20.-y, 11.10.-z, 63.75.+z, 64.60.Cn

\section{INTRODUCTION}

The double-well $\phi^{4}$ model in $1+1$ dimensions has been extensively studied in the context of symmetry breaking transitions. As is well-known, the equilibrium classical statistical mechanics of a $1+1$ dimensional field theory reduces to a time-independent quantum mechanics problem via the transfer integral method [1,2]. However, the Schrödinger equation with a $\phi^{4}$ potential does not have any known exact solutions. To overcome this problem, we turn to another double-well system that is quasi-exactly solvable (QES), where by a QES problem we mean one in which the exact partial diagonalization of the Hamiltonian may be carried out [3]. Knowing just the low-lying eigenvalues and eigenfunctions is sufficient to obtain almost complete information regarding the classical thermodynamics of the field theoretic system. As will be seen below the QES property holds only at a discrete set of temperatures. Our strategy is to tune system parameters so as to straddle an interesting region in temperature space which, for this paper, we have taken to be the region around the short-range order ("kink") transition point.

An important motivation for exact results is validation of numerical techniques: recent advances in large scale computation have made it possible to calculate quantities such as the probability distribution function (PDF) us-

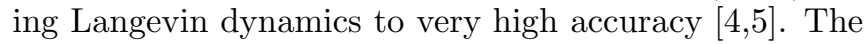
PDF is the probability distribution of field values averaged over the total system volume. Alternatively, it is equal to the square of the ground state wavefunction of the transfer operator at a given temperature. All thermodynamic information can be shown to reside in the PDF [6, 7]. Calibration against the PDF calculated from the exact ground state wavefunction is an essential guide when performing error and convergence analysis of the Langevin equation [8].

The double-well QES system that we focus on in this paper is specified by the double sinh-Gordon potential $V(\phi)=(\zeta \cosh 2 \phi-n)^{2}$, where $\zeta$ is a positive parameter and $n$ is a positive integer $[5,9,10,11,12,13,14$. This potential is the hyperbolic analog of the double sine-Gordon system [15]. Similar potentials arise in the context of the quantum theory of molecules (e.g., a homonuclear diatomic molecule), wave motion describing the normal modes of vibration of a stretched membrane of variable density [16], and as the solution of a Fokker-Planck equation [9]. Note that the hyperbolic analog of the sineGordon equation is a single well potential (sinh-Gordon [17]) and thus uninteresting from the soliton statistical mechanics perspective.

Below we find exact solutions for the ground and a few excited state energy eigenvalues and wavefunctions for certain temperatures both above and below the shortrange transition temperature. This allows analytic calculation of the PDF and correlation functions $\left(C_{1}\right.$ and $C_{2}$ ) and direct comparison with those calculated from a Langevin simulation. The analytic kink profile can also be compared with the corresponding field configuration in the simulation. We find striking agreement 
between the exact results and simulations. The proven high quality of the simulations provides an important validation for PDF-based thermodynamics implemented via Langevin methods.

Before proceeding to a more detailed view of the DSHG model we contrast its main features with those of other models that have been used previously for analytic studies. The Schrödinger equation with a double-quadratic potential $V(\phi)=\frac{1}{2}(|\phi|-1)^{2}$ [18,19 is in fact exactly solvable. However, the energy eigenvalues are not known as simple functions but may be obtained only as a solution of transcendental equations involving parabolic cylinder functions. Moreover, this potential is not a smooth function and has a cusp at $\phi=0$. Other smoothly varying double-well potentials, e.g., the Manning potential $V(\phi)=-A \operatorname{sech}^{2}(\phi / 2 \rho)+B \operatorname{sech}^{4}(\phi / 2 \rho)$ [20] and the double-Gaussian model $V(\phi)=1 / 2(\phi / \rho)^{2}-$ $\ln \cosh \left(\phi \sigma / \rho^{2}\right)$, 21] do not allow exact or quasi-exact solvability of the associated Schrödinger equation. We note that the double-Morse potential, which arises in several physical contexts such as hydrogen-bonded chains 22], is closely related to the DSHG potential.

\section{THE DOUBLE SINH-GORDON MODEL}

We start with the continuum representation of a model Hamiltonian describing a system capable of undergoing a displacive transition:

$$
H=\int \frac{d x}{l}\left[\frac{m}{2} \phi_{t}^{2}+V(\phi)+\frac{m c_{0}^{2}}{2} \phi_{x}^{2}\right],
$$

where $l$ is the lattice spacing, $m$ the mass of particles (ions) and $c_{0}$ the velocity of low-amplitude sound waves (phonons) in the associated discrete problem [2]. Here the potential is

$$
V_{D S H G}(\phi)=(\zeta \cosh 2 \phi-n)^{2},
$$

where $\zeta$ is a positive parameter. While the value of $n$ is not restricted in principle, it has to be a positive integer for the QES property to hold, along with $n>\zeta$ in order to have a double well. The two minima occur at $\cosh 2 \phi_{0}=n / \zeta$ with $V_{\text {min }}=0$ and a local maximum at $\phi=0$ with $V(0)=(n-\zeta)^{2}$. We depict the potential for a few values of $n$ and $\zeta$ in Fig. 1 .

The DSHG potential written in the form (2) has all the generic features of a double-well potential such as Landau-Ginzburg, but allows for much greater analytic progress. Below we find exact solutions for (1) a kink, (2) a kink lattice, (3) phonon dispersion, and (4) the first few eigenvalues and eigenfunctions of the transfer operator at certain temperatures both above and below the transition, thereby allowing analytic calculation of the PDF and correlation functions in the thermodynamic limit.

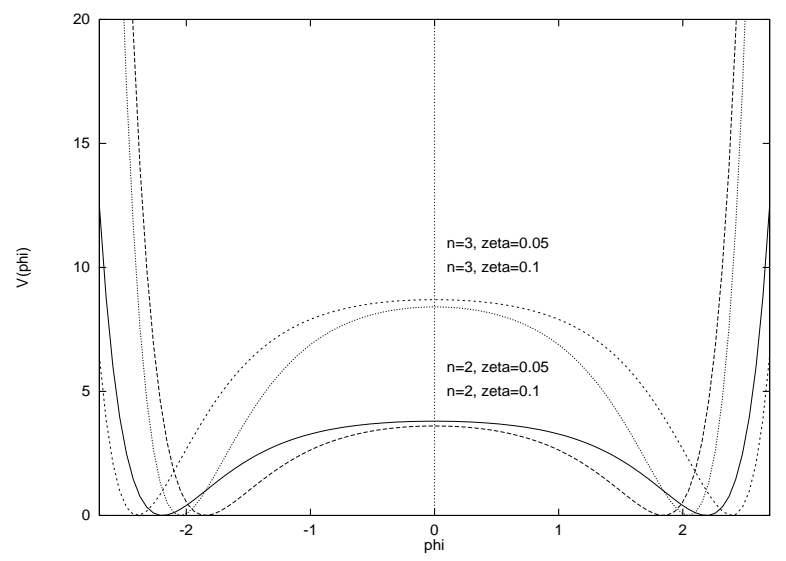

FIG. 1. The DSHG potential for $n=2,3$ and $\zeta=0.05,0.1$.

\section{KINK AND KINK LATTICE SOLUTIONS}

The single kink solution for the double sinh-Gordon potential is nothing but the finite energy solution of the equation of motion $\frac{1}{2} g \phi_{x}^{2}(x)=V_{D S H G}(\phi)$. The kink/antikink located at $x_{0}$ is given by [5, 10.

$$
\begin{aligned}
\phi(x) & = \pm \tanh ^{-1}\left(\sqrt{\frac{n-\zeta}{n+\zeta}} \tanh \frac{x-x_{0}}{\xi}\right) ; \\
\xi & =\sqrt{\frac{g}{2\left(n^{2}-\zeta^{2}\right)}} ; \quad n>\zeta .
\end{aligned}
$$

Here $g=m c_{0}^{2}$ and $\tanh \phi_{0}=\sqrt{(n-\zeta) /(n+\zeta)}$. The constant $g$ is often introduced in condensed matter treatments as a phenomenological parameter and controls the kink size. Note that instead of the potential (2) one could consider a more general potential of the form $a(\zeta \cosh 2 b \phi-n)^{2}$. It is easily seen that the kink solution (3) is unchanged except for the replacement of $g$ by $g / a b^{2}$. For simplicity, throughout this paper we take $a, b=1$ since solutions for the general case are trivially obtained from here. Traveling kink solutions are obtained by boosting to velocity $v$ via $x \rightarrow\left(1-v^{2}\right)^{-1 / 2}(x-v t)$. The total energy density $\epsilon(x)=V(\phi)+\frac{1}{2} \phi_{x}^{2}$. Thus, the energy (or rest mass) of a kink is

$$
\begin{aligned}
E_{s}= & \int_{-\infty}^{\infty} \epsilon(x) d x \\
& =4 \xi n \sqrt{n^{2}-\zeta^{2}}\left(\tanh ^{-1} \sqrt{\frac{n-\zeta}{n+\zeta}}\right)-2 \xi\left(n^{2}-\zeta^{2}\right)
\end{aligned}
$$

The topological charge of the kink is

$$
\begin{aligned}
Q & =\int_{-\infty}^{\infty} \frac{\partial \phi}{\partial x} d x=\phi(+\infty)-\phi(-\infty) \\
& =2 \tanh ^{-1} \sqrt{\frac{n-\zeta}{n+\zeta}}=2 \phi_{0} .
\end{aligned}
$$


The kinetic energy contribution of a moving kink with velocity $v$ is obtained from $\phi\left((x-v t) / \xi \sqrt{1-v^{2}}\right)$

$$
\begin{aligned}
& E_{k i n}=\int_{-\infty}^{\infty} d x \frac{m}{2} \phi_{t}^{2}=\frac{m^{*}}{2} v^{2} ; \\
& m^{*}=\frac{m}{\xi \sqrt{1-v^{2}}}\left(\frac{2 n}{n-\zeta} \sqrt{\frac{n+\zeta}{n-\zeta}} \tanh ^{-1} \sqrt{\frac{n-\zeta}{n+\zeta}}-1\right) .
\end{aligned}
$$

To calculate the contribution to the internal energy and specific heat from kinks we treat them as a onedimensional gas of indistinguishable, independent, static particles of width $\sim 2 \xi$. The free energy contribution is then given by

$$
\begin{aligned}
& F_{K}= \\
& -N k_{B} T \frac{l}{\xi}\left(1+\frac{1}{2} \ln \frac{2 \pi k_{B} T}{m^{*} B^{2}}\right) \exp \left(-\frac{E_{s}}{k_{B} T \sqrt{1-v^{2}}}\right),
\end{aligned}
$$

where $B$ is a phase space normalization constant.

In order to understand kink-antikink interactions, it is useful to construct a kink lattice solution which is obtained by twice integrating the equation of motion

$$
\pm \sqrt{\frac{2}{g}}\left(x-x_{0}\right)=\int_{\phi\left(x_{0}\right)}^{\phi(x)} \frac{d \phi}{\sqrt{V(\phi)-V_{0}}}
$$

with the boundary conditions for a finite length $L=x_{2}-$ $x_{1}: \phi_{x}\left(x_{1}\right)=\phi_{x}\left(x_{2}\right)=0$, and $V\left(\phi\left(x_{1}\right)\right)=V\left(\phi\left(x_{2}\right)\right)=$ $V_{0}$. Here $V_{0}$ and $x_{0}$ are integration constants and $V(x)>$ $V_{0}$ for $x \in\left(x_{1}, x_{2}\right)$. The solution is

$$
\begin{aligned}
\phi_{L}(x) & = \pm \tanh ^{-1}\left(\tanh \phi_{1} \operatorname{sn}\left(\frac{x-x_{0}}{\xi_{L}}, k\right)\right) \\
k & =\frac{\tanh \phi_{1}}{\tanh \phi_{2}} ; \quad d=4 K \xi_{L} \\
\xi_{L} & =\frac{k}{2 \zeta \sinh \phi_{1} \cosh \phi_{2}} \sqrt{\frac{g}{2}}
\end{aligned}
$$

where $d$ is the periodicity of the kink lattice, $K(k)$ is the complete elliptic integral of the first kind with modulus $k, \operatorname{sn}(x, k)$ is the Jacobi elliptic function, and

$$
\begin{aligned}
\cosh 2 \phi_{1,2}= & \frac{n}{\zeta} \mp \frac{\sqrt{V_{0}}}{\zeta} ; \quad k^{2}=\frac{n^{2}-\left(\sqrt{V_{0}}+\zeta\right)^{2}}{n^{2}-\left(\sqrt{V_{0}}-\zeta\right)^{2}} \\
& 0<V_{0}<V(\phi=0)=(\zeta-n)^{2}
\end{aligned}
$$

Equivalently,

$$
\begin{aligned}
& \tanh ^{2} \phi_{1,2}= \\
& \frac{n-\zeta}{n+\zeta}\left[\frac{\left(1+k^{2}\right) \zeta \mp k^{\prime 2}(n+\zeta)+\sqrt{k^{4} n^{2}+4 k^{2} \zeta^{2}}}{\left(1+k^{2}\right) \zeta \mp k^{\prime 2}(n-\zeta)+\sqrt{k^{\prime 4} n^{2}+4 k^{2} \zeta^{2}}}\right]
\end{aligned}
$$

and the characteristic length of a kink in the lattice

$$
\begin{aligned}
& \xi_{L}^{2}=\frac{g}{2}\left\{-n^{2}\right. \\
& \left.\zeta^{2}\left[\frac{\left(1+k^{2}\right) \zeta^{2}-k^{\prime 2}\left(n^{2}-\zeta^{2}\right)+\zeta \sqrt{k^{\prime 4} n^{2}+4 k^{2} \zeta^{2}}}{\left(1+k^{2}\right) \zeta^{2}+\zeta \sqrt{k^{\prime 4} n^{2}+4 k^{2} \zeta^{2}}}\right]^{2}\right\}^{-1}
\end{aligned}
$$

where $k^{\prime}=\sqrt{1-k^{2}}$ is the complementary modulus.

The topological charge (per period) in the lattice problem $Q_{L}=2 \phi_{L}(K)=2 \phi_{1}$ is smaller than the single kink case $Q=2 \phi_{0}=2 \tanh ^{-1} \sqrt{(n-\zeta) /(n+\zeta)}$. Similarly, the kink size in the kink lattice, $\xi_{L}$, is also smaller than the free kink size $\xi$.

The energy of the kink lattice per period (i.e., the energy per kink-antikink pair plus the interaction energy) is obtained, after considerable algebra, using $\phi_{L}(x)$ in Eq. (1) and evaluating various integrals involving Jacobi elliptic functions 23]:

$E_{L}=4 \xi_{L}\left[(n+\zeta)^{2} K+\frac{\xi^{2}}{\xi_{L}^{2}}\left(n^{2}-\zeta^{2}\right)(K-E)-4 n \zeta \Pi\right]$

where $E(k)$ and $\Pi\left(\tanh ^{2} \phi_{1}, k\right)$ are complete elliptic integrals of second and third kind, respectively. Note that in the dilute limit $(k \rightarrow 1, d \rightarrow \infty)$ the divergences in $K(k)$ and $\Pi\left(\tanh ^{2} \phi_{1}, k\right)$ exactly cancel out and we recover the single kink result $E_{s}$. The interaction energy as a function of separation (i.e., $k$ or $d$ ) is given by $E_{i n}=E_{L}-2 E_{s}$.

The repulsive (attractive) kink-kink (kink-antikink) interaction in the asymptotic limit can be calculated by letting $k \rightarrow 1$ or $k^{\prime} \rightarrow 0$ and expanding $E_{L}$ up to order $k^{\prime 4}$. However, for the DSHG model it can directly be obtained from the asymptotic form of the single kink solution by using Manton's formula [24],

$$
U(r)= \pm \frac{2}{\xi} \frac{n^{2}-\zeta^{2}}{\zeta^{2}} \exp \left(-\frac{d}{\xi}\right)
$$

\section{KINK LATTICE THERMODYNAMICS}

One can compute various "thermodynamic" quantities associated with the kink lattice similar to the sineGordon case [25]. The internal energy per kink $U / N=$ $E_{L} / 2$ and $L / N=d$ imply that the thermodynamic pressure

$$
\begin{aligned}
& P=-\left(\frac{\partial U}{\partial L}\right)_{T=0}= \\
& \frac{1}{2}\left[2 n \frac{\xi}{\xi_{L}}\left(n^{2}-\zeta^{2}\right)^{1 / 2} E(\beta, k)-\frac{\xi^{2}}{\xi_{L}^{2}}\left(n^{2}-\zeta^{2}\right)-(n-\zeta)^{2}\right]
\end{aligned}
$$

where $\beta=\sin ^{-1} \tanh \phi_{2}$. The enthalpy is $H=(U+$ $P L)=N \mu$, with the chemical potential given by

$$
\mu=2 E\left(n^{2}-\xi^{2}\right) \frac{\xi^{2}}{\xi_{L}^{2}}\left[\frac{2 n \xi_{L}}{\xi\left(n^{2}-\zeta\right)^{1 / 2}} F(\beta, k)-1\right]
$$


where $F(\beta, k)$ and $E(\beta, k)$ are incomplete elliptic integrals of the first and second kind, respectively. Similarly, the isothermal compressibility $H_{T}=-(1 / L)(\partial L / \partial P)_{T}$ can be calculated:

$$
\begin{aligned}
H_{T}= & \frac{1}{2}\left\{1+\left[\xi_{L} / k\left(\partial \xi_{L} / \partial k\right)\right]\left[E / k^{\prime 2} K-1\right]\right\} \\
& \times\left\{\left(n \xi / \xi_{L}\right) \sqrt{n^{2}-\zeta^{2}} E(\beta, k)-\left(n^{2}-\zeta^{2}\right)\left(\xi^{2} / \xi_{L}^{2}\right)\right. \\
& -(n \xi / k) \sqrt{n^{2}-\zeta^{2}}(\partial \beta / \partial k)\left(\partial \xi_{L} / \partial k\right) \\
& \times\{E(\beta, k)-F(\beta, k)\}\}^{-1}
\end{aligned}
$$

where $\partial \beta / \partial k=\cosh \phi_{2}\left(\partial \tanh \phi_{2} / \partial k\right)$ and $\partial \xi_{L} / \partial k$ can be obtained from the above expressions for $\tanh \phi_{2}$ and $\xi_{L}$ as a function of $k$.

\section{STABILITY OF KINKS}

Since the transformation $\phi \rightarrow \tanh \phi$ connects the equation of motion (see below) for the $\phi^{4}$ and DSHG models, the linear stability of the kink and kink lattice solutions in the two models is directly related. We find that the DSHG kink stability equation is the Heun's equation which has at least two bound states: (1) the usual zero frequency Goldstone mode and (2) a kink shape oscillation mode. Similarly, for linear stability of the kink lattice we can write $\tanh \left(\phi_{L}, t\right)=$ $\tanh \left(\phi_{L}\right)+\phi_{L}(x, t)$. Assuming a harmonic time variation $\phi_{L}(x, t)=\psi(x) \exp (i \omega t)$ we find that the kink lattice stability equation is also Heun's equation. A special case is the Lamé equation of order two $(\nu=2)$ which arises in the context of the stability of the $\phi^{4}$ model kink lattice solution:

$$
\psi_{x x}+\left[A(\omega, k)-\nu(1+\nu) k^{2} s n^{2} x\right] \psi=0 .
$$

The solutions of this equation are the Lamé functions [26] with $2 \nu+1$ eigenvalues. This implies a maximum of five bound states. Note that in the integrable case of the sine-Gordon equation one gets the Lamé equation of order one $(\nu=1)$ implying a maximum of three bound states for the kink lattice and only the Goldstone mode for the kink [25]. However, the DSHG Heun's equation does not reduce to a Lamé equation. These results apply to linear stability (kink-phonon scattering) and cannot be readily generalized to the case of nonlinear stability.

\section{PHONONS}

The equation of motion for the field is given by

$$
m \phi_{t t}-\phi^{\prime \prime}+2 \zeta[\zeta \sinh 4 \phi-2 n \sinh 2 \phi]=0 .
$$

This equation can be linearized around (1) $\phi=0$ or (2) $\phi=\phi_{0}$ leading to higher and lower energy phonons, respectively. For phonons around $\phi=0$ the above equation can be approximated by

$$
m \phi_{t t}-\phi^{\prime \prime}+\left(8 \zeta^{2}-8 n \zeta\right) \phi+\frac{16}{3}\left(4 \zeta^{2}-n \zeta\right) \phi^{3}=0 .
$$

For small amplitude oscillations $(\phi \ll 1)$ we retain only the linear term. Assuming $\phi(x, t)=\bar{\phi} \exp \left[i\left(q x-\omega_{q} t\right)\right]$ we get the dispersion relation

$$
\omega_{q}^{2}=q^{2}-8 \zeta(n-\zeta),
$$

where the frequency $\omega_{q}$ will be real only for finite $q^{2} \geq$ $8 \zeta(n-\zeta)$. Of course, for $n<\zeta$ (when $\phi=0$ is the minium of the potential), the frequency $\omega_{q}$ is always real.

Similarly, the phonon dispersion around the minima $\pm \phi_{0}$ for this model is

$$
\omega_{q}^{2}=q^{2}+8\left(n^{2}-\zeta^{2}\right) .
$$

Note that the frequencies are real for all $q \geq 0$ (and $n>\zeta)$ and the quantity $4\left(n^{2}-\zeta^{2}\right)$ is equivalent to the coefficient $|A|$ of the quadratic term in the corresponding $\phi^{4}$ model [2]. The associated phonon contribution to the free energy is

$$
F_{v i b}=\frac{1}{2 \pi \delta} \ln \left(\frac{2 \pi}{\delta \beta}\right)+\frac{1}{\beta} \sqrt{2\left(n^{2}-\zeta^{2}\right)},
$$

with $\delta$ being the lattice constant and $\beta \equiv 1 / k_{B} T$. The contribution to the internal energy and specific heat from phonons and kinks can be directly calculated using $F_{v i b}$ and $F_{K}$, respectively. However, here we do not consider the contribution to the free energy due to kink-phonon interactions [19].

\section{TRANSFER OPERATOR FORMALISM}

The transfer operator method transforms the problem of finding the canonical partition function for a system to an exactly equivalent problem of finding the eigenvalues of a certain integral operator. In the continuum limit, this problem can be further reduced to finding the energy eigenvalues of a related quantum mechanical problem (i.e., a Schrödinger equation with an effective potential). In addition, the transfer operator formalism also provides exact expressions for correlation functions. The key property of the DSHG potential (2) is that the $n$ lowest eigenvalues and wavefunctions are known exactly, in contrast to other QES systems. This enables exact evaluation of the PDF and the correlation functions $C_{1}$ and $C_{2}$ (see below) at several temperatures. Aside from validation of Langevin results against these quantities, the exact single kink solution can be checked against that obtained from the low temperature Langevin field configuration.

Turning now to the computation of $Z_{c l}$, we note that this calculation can be divided into two parts: a trivial Gaussian integration over the field momentum, and 
a computation of the configurational partition function, which via the transfer integral method becomes equivalent to solving a Schrödinger-like equation [1]. The Hamiltonian for the DSHG theory is

$$
H=\int d x\left[\frac{1}{2} \pi^{2}+\frac{1}{2} \phi_{x}^{2}+V_{D S H G}(\phi)\right]
$$

and this leads to the Schrödinger-like equation for the eigenvalues and eigenfunctions of the transfer operator,

$$
-\frac{1}{2 \beta^{2}} \frac{\partial^{2}}{\partial \phi^{2}} \Psi_{k}+(\zeta \cosh 2 \phi-n)^{2} \Psi_{k}=E_{k} \Psi_{k} .
$$

This is a QES system for which, at $2 \beta^{2}=1$, using results for a related potential from Ref. [9], the eigenstates of the first $n$ levels can be found for $n=1,2,3,4$. (We have been able to extend this to the cases $n=5,6$.) However, what one really wants is to consider a given fixed- $n$ theory and obtain eigenstates at different temperatures. It is easy to see from Eq. (16), by simple rescaling, that solutions of a fixed- $n$ theory at certain values of $\beta$ are the same as the solutions of another theory (different $n$ and $\zeta$ ) at $2 \beta^{2}=1$. Depending on the chosen value of $n$, exact solutions are available at different fixed values of $\beta$. Here, we restrict ourselves to one such family $(n=2)$ which allows the exact computation of the first few eigenstates at $8 \beta^{2}=$ $m^{2}(m=1, \cdots, 6)$. For illustration, three examples of the (unnormalized) ground states are given below (see also Fig. 2). The first (high temperature, $\beta^{2}=1 / 8$ ) has an eigenfunction with a single peak while the other two (lower temperatures, $\beta^{2}=1 / 2$ and $9 / 8$ ) have a double peak:

$$
\begin{aligned}
\left.\Psi_{0}(\phi)\right|_{\beta^{2}=\frac{1}{8}}= & \exp \left(-\frac{1}{4} \zeta \cosh 2 \phi\right) \\
\left.\Psi_{0}(\phi)\right|_{\beta^{2}=\frac{1}{2}}= & \cosh \phi \exp \left(-\frac{1}{2} \zeta \cosh 2 \phi\right) \\
\left.\Psi_{0}(\phi)\right|_{\beta^{2}=\frac{9}{8}}= & {\left[3 \zeta+\left(1+\sqrt{1+9 \zeta^{2}}\right) \cosh 2 \phi\right] } \\
& \times \exp \left(-\frac{3}{4} \zeta \cosh 2 \phi\right)
\end{aligned}
$$

with corresponding ground state energies, $E_{0}=4+\zeta^{2}$, $E_{0}=\zeta^{2}-2 \zeta+3$ and $E_{0}=\zeta^{2}+\frac{28}{9}-\frac{8}{9} \sqrt{1+9 \zeta^{2}}$ respectively. The PDF for the classical field $\phi$ is just the square of the normalized ground state eigenfunctions. Solutions at higher energies and other values of $\beta$ are given in Appendix A.

Once the eigenvalues of the transfer operator are known, they can be used to compute the correlation functions $C_{1}(x)=\langle\phi(0) \phi(x)\rangle$ and $C_{2}=\left\langle\delta \phi^{2}(0) \delta \phi^{2}(x)\right\rangle$, using

$$
\begin{aligned}
& C_{1}(x)=\sum_{k}\left|\left\langle\Psi_{k}|\phi| \Psi_{0}\right\rangle\right|^{2} \exp \left[-\beta|x|\left(E_{k}-E_{0}\right)\right], \\
& C_{2}(x)=\sum_{k}\left|\left\langle\Psi_{k}\left|\delta \phi^{2}\right| \Psi_{0}\right\rangle\right|^{2} \exp \left[-\beta|x|\left(E_{k}-E_{0}\right)\right] .
\end{aligned}
$$

It is apparent that at large distances, $C_{1}$ and $C_{2}$ are dominated by the lowest state with nonvanishing matrix elements: the first excited state in the case of $C_{1}$ and the second excited state in the case of $C_{2}$. Since $E_{0}, E_{1}$, and $E_{2}$ are known at certain temperatures, the large distance behavior of these correlation functions can be found exactly and compared with the results from simulations. Static structure factors may also be calculated in much the same way.

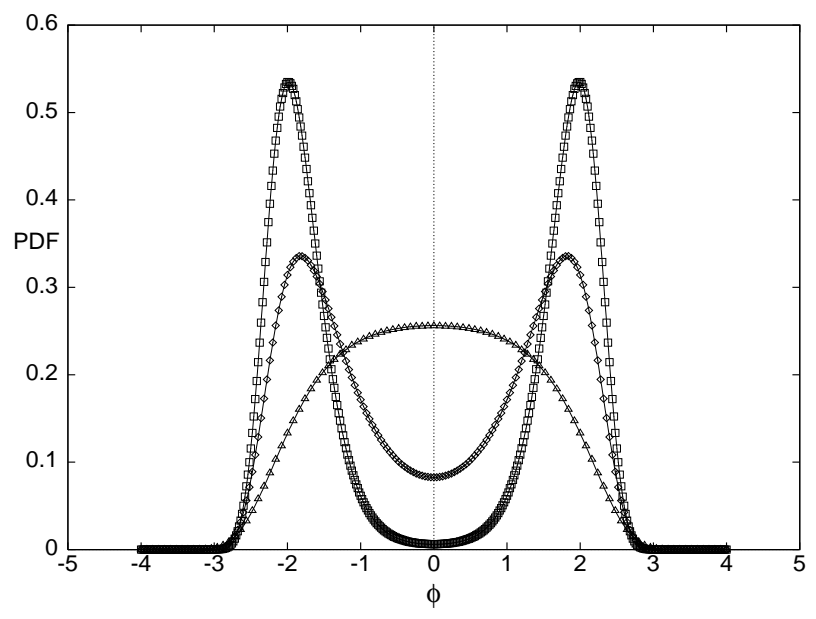

FIG. 2. PDFs at three values of $\beta^{2}$ with the continuum exact solutions shown as solid lines. Results from Langevin simulations are superimposed at the $\beta$ values: $1 / 8$ (triangles), $1 / 2$ (diamonds), and $9 / 8$ (squares).

At this point, it is important to mention the connection between the "quantum" calculations and kink physics. In the context of kink statistical mechanics, it is usual to introduce a phenomenological description of kinks as particles in a grand canonical ensemble. However, this is unnecessary, and all such thermodynamical information can be extracted directly from the Schrödinger description of the transfer operator. For example, the kink density has been obtained in this way in Ref. 27. Simpler quantities like $C_{1}$ and $C_{2}$ have obvious natural interpretations in terms of kinks. The $C_{1}$ correlation length is related to the kink/antikink spacing and increases monotonically as $\beta$ increases (Fig. 3). The behavior of the $C_{2}$ correlation length is more subtle, since $C_{2}$ is not sensitive to domain size. At both high temperatures (no kinks) and low temperatures (number of kinks exponentially suppressed), the correlation length is dominated by the thermal phonon contribution. However, in the range of temperatures close to the kink transition the fluctuations on the kink length scale become important and can dominate $C_{2}$. At these temperatures one might expect a maximum in the $C_{2}$ correlation length, on the order of the kink size, and this is indeed what we observe numerically (Fig. 4). The Schottky anomaly in the specific heat [27] arises for the very same reason. 


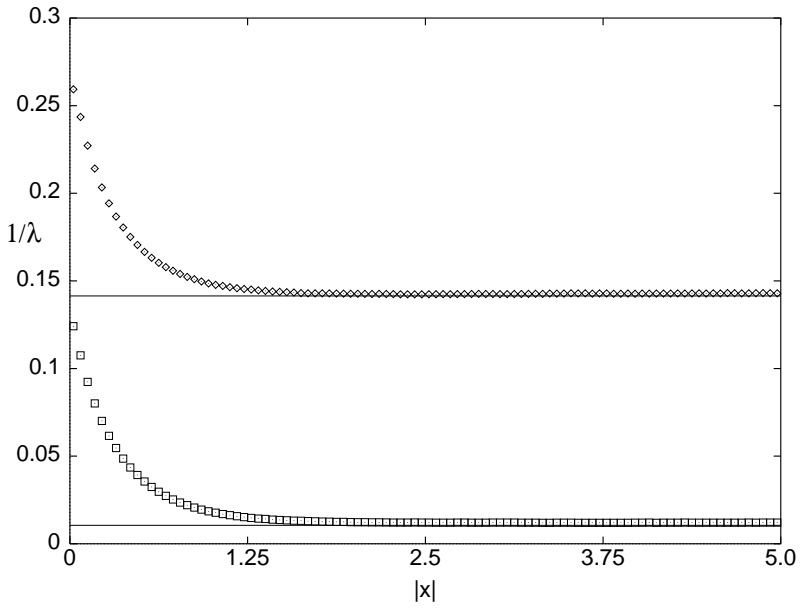

FIG. 3. Comparison of the inverse correlation lengths from $C_{1}(x)$ obtained via Langevin simulations $\left[\beta^{2}: 1 / 2\right.$ (diamonds), and $9 / 8$ (squares)] and from the large $|x|$ continuum exact results (solid lines).

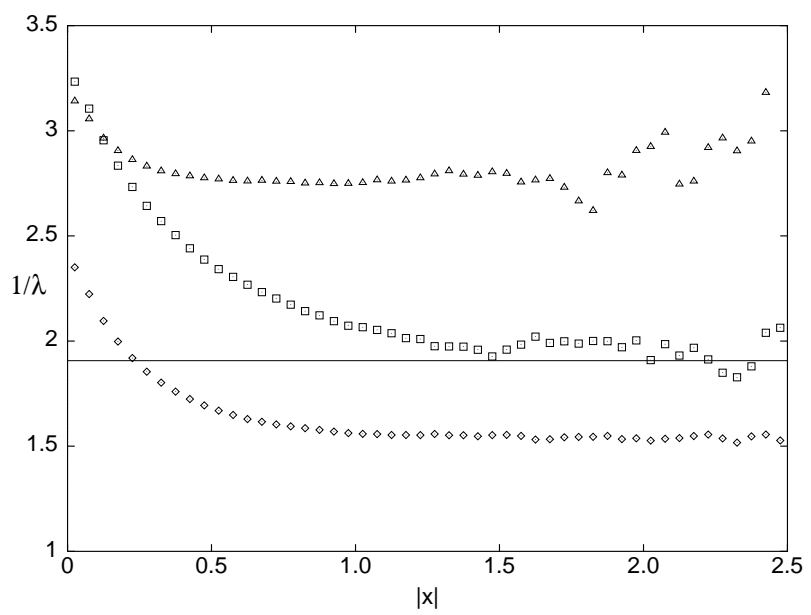

FIG. 4. The numerical and exact inverse correlation lengths from $C_{2}(x)$ are compared for the same three temperatures and with the same conventions as in Fig. 2. The solid line is the large $|x|$ continuum exact result for $\beta^{2}=9 / 8$. The largest correlation length is at the intermediate value of $\beta$ (see text).

\section{QES SOLUTIONS}

The explicit QES solutions (exact eigenvalues and eigenfunctions) for $2 \beta^{2}=1$ and $n=1,2,3,4$ are given by Razavy [9] while we have been able to extend these results to $n=5,6$. We now explain in more detail how to relate these results to a theory with a given value of $n$ (say $n=2$ ) but with different values of $\beta$. We start from the Schrödinger equation (16) at $2 \beta^{2}=1$ and arbitrary $n(=1,2, \cdots, 6)$. On multiplying both sides of this equation by $4 / n^{2}$, it immediately follows that the exact solutions for a given value of $n, \zeta, E$ at $2 \beta^{2}=1$ are equivalent to the solution of the DSHG equation at $n=2$ but at $8 \beta^{2}=n^{2}, \hat{E}=4 E / n^{2}, \hat{\zeta}=2 \zeta / n$. Clearly, instead of fixing $n=2$, one could choose any of the six values of $n$ and for each case one knows the exact solutions for that theory at six different temperatures. As an illustration, the explicit QES solutions (exact eigenvalues and eigenfunctions) for $n=1,2$ at six different temperatures are presented in Appendix A.

\section{CONNECTION WITH $\phi^{4}$ AND DOUBLE SINE-GORDON MODELS}

As a final point, we consider the relationship of the DSHG theory to the more familiar Landau-Ginzburg model. Scrutiny of the static equation of motion $\left[\phi_{x}^{2}=\right.$ $V(\phi)]$ reveals an important connection between the kink (and kink lattice) solutions of the $\phi^{4}$ model and the double sine-Gordon (DSG) and DSHG models. Consider the $\phi^{4}$ potential $V_{4}(u)=\left[(n+\zeta) u^{2}-(n-\zeta)\right]^{2}$. The substitution $u=\tanh \phi$ takes the (static) equations of motion over to the DSHG equations. The alternative substitution $u=\tan \phi$ leads to a DSG model. This means that all known solutions of the $\phi^{4}$ equation of motion can be directly taken over to the DSHG and DSG equations of motion (and vice versa). As one use of this interesting relationship, the DSG kink lattice solution (not known heretofore in the literature) can be written down directly in case $V_{D S G}=(\zeta \cos 2 \phi-n)^{2}$ :

$$
\phi_{L}= \pm \tan ^{-1}\left(\tan \phi_{1} \operatorname{sn}\left(\frac{x-x_{0}}{\xi_{L}}, k\right)\right)
$$

simply by using the substitution $\tanh \phi \rightarrow \tan \phi$ in the kink lattice solution $\phi_{L}$ of the DSHG model.

This connection enables us to write down by inspection not just the kink solutions but their total energy as well, which is often a very tedious task. Similarly, knowing the linear stability of the $\phi^{4}$ kink and kink lattice, important results follow for the stability of corresponding solutions of the DSG and DSHG models (see Sec. V). Moreover, since we know that the DSHG model is an example of a QES system, and considering the very similar way in which the DSG and DSHG models are related to $\phi^{4}$, it is logical to conjecture that some DSG model may also be a QES system. Indeed, this is the case, and we have found several exact eigenvalues and eigenfunctions at many temperatures for a particular DSG system. The exact statistical mechanical results for that model, similar to the DSHG results presented here, will be reported elsewhere [32].

Alternatively, if we start from the $\phi^{4}$ potential $V_{4}(u)=$ $\left[(n+\zeta) u^{2}+(n-\zeta)\right]^{2}$, the transformation $u=\tan \phi$ takes the (static) equations of motion over to the model $V_{D S G}=[\zeta \cos 2 \phi-n]^{2}$. For $n<\zeta$ we get the doubly periodic DSG potential with two types of kinks ("small" and "large"). When $n>\zeta$, we get the singly periodic DSG potential with the $2 \pi$-kink solution (see Fig. 1 of 
Ref. [28]). The associated $V_{4}(u)$ in this case is a single well potential. However, the real transformation does not lead to a QES DSG system unlike the transformation above.

We note that the transformations $u=\tan \phi$ and $u=\tanh \phi$ connect the equation of motion of the $\phi^{4}$ model to that of the DSG and DSHG systems, respectively. On the other hand, the transformations $u=\cos \phi$ and $u=\cosh \phi$ connect the equation of motion of the $\phi^{4}$ model to that of the exactly solvable sine-Gordon $\left(V_{S G}=\right.$ $\left.\sin ^{2} \phi\right)$ and sinh-Gordon $\left(V_{S H G}=\sinh ^{2} \phi\right)$ systems, respectively, provided we start with $V_{4}(u)=\left(u^{2}-1\right)^{2}$. We also note that the number of periodicity in the trigonometric potentials (DG, DSG, triple sine-Gordon (TSG), ...) corresponds to the number of wells in the associated hyperbolic potentials (SHG, DSHG, triple sinh-Gordon (TSHG), ...). We conjecture that the equations of motion for the TSG and TSHG models can be directly connected with that of the $\phi^{6}$ model via appropriate transformations. In addition, while the TSG equation [15, 29] is known to arise in nonlinear optics the TSHG potential [e.g. $V_{T S H G}=A \cosh 2 \phi+B \cosh 4 \phi+C \cosh 6 \phi$ ] can serve as a model system to study first-order phase transitions.

\section{SIMULATION RESULTS}

The numerical study of kink statistical mechanics was carried out via Langevin simulations. The advantage of such simulations is that representative field configurations (see Fig. 5) are available and can be analyzed to compute such quantities as the kink density, finite temperature kink profile, the spatial kink distribution, kink transport and nucleation, etc. This sort of analysis is not possible with Monte Carlo techniques.

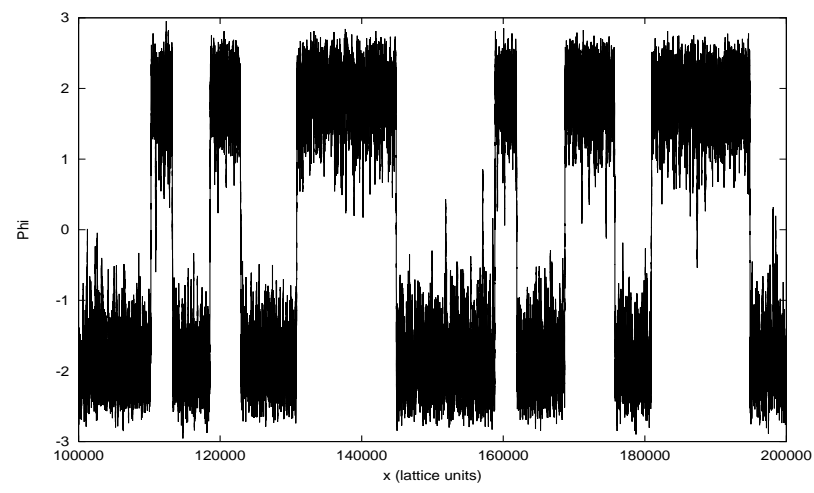

FIG. 5. Sample kink configurations from a $100 K$ section of a $400 K$ unit simulation with $\beta^{2}=9 / 8$.

The additive noise Langevin equation for the double sinh-Gordon model is

$\partial_{t t}^{2} \phi=\partial_{x x}^{2} \phi-\eta \partial_{t} \phi-4 \zeta(\zeta \cosh 2 \phi-n) \sinh 2 \phi+\hat{F}(x, t)$ where the viscosity $\eta$ and the Gaussian white noise $\hat{F}$ are related by the fluctuation-dissipation theorem:

$$
\left\langle\hat{F}(x, t) \hat{F}\left(x^{\prime}, t^{\prime}\right)\right\rangle=2 \eta \beta^{-1} \delta\left(x-x^{\prime}\right) \delta\left(t-t^{\prime}\right) .
$$

The lattice versions of the above continuous equations (with periodic boundary conditions) were solved using standard techniques [30]. Random initial conditions were driven to equilibrium and the results sampled in time thereafter to yield time averaged PDFs, etc. The use of the Langevin technique for obtaining thermodynamic quantities is straightforward and as shown below, can be remarkably accurate. The availability of exact solutions is very useful since they set stringent criteria for accuracy that must be met by numerical methods. In the simulations reported below we used a lattice size of typically $512 K$ points with a lattice spacing $\Delta=0.025$. The time step was taken to be $\epsilon=0.005$. [We employed both $\sqrt{\epsilon}$ (Euler) and $\epsilon^{2}$ (Runge-Kutta) order stochastic integration algorithms.] These values are substantially smaller than what has been the norm so far in Langevin simulations of other $1+1$ dimensional field theories [31]. In fact, based on our experience with the double sinh-Gordon system it is likely that errors in previous Langevin analyses have been as high as $30 \%$.

Fig. 2 shows the striking agreement between the numerically obtained and the exact continuum PDFs at three temperatures: The worst case departure is at the level of parts per thousand. The comparisons for the inverse correlation lengths are given in Figs. 3 and 4. DSHG system parameters are $n=2, \zeta=0.05$. For $C_{1}$, the numerical values are $1 / \lambda=0.1425\left(\beta^{2}=1 / 2\right)$ and $1 / \lambda=0.012\left(\beta^{2}=9 / 8\right)$ as compared to the exact values in the continuum theory of 0.14142 and .0105 , respectively. The small offset between the continuum and lattice calculations is due to the finite value of the lattice constant and is consistent with estimates from higherorder contributions to the transfer integral [8]. The kink number density at low temperatures is related to the correlation length via $N_{k} \simeq 1 / 4 \lambda$ 27]. This general relationship is borne out in the DSHG simulations.

The high quality of these numerical simulations implies that the PDF can now be used directly to compute thermodynamic quantities at any temperature. Since the PDF is just the square of the ground state wave function of the Schrödinger equation, one can use it to compute the ground state energy $E_{0}$ numerically, from which the internal energy $\left(U=\partial E_{0} / \partial \beta\right)$, the free energy $\left(F=E_{0} / \beta\right)$, and the entropy $\left(S=\beta \partial E_{0} / \partial \beta-E_{0}\right)$ can all be computed in a straightforward way. The specific heat involves two $\beta$ derivatives and is difficult to obtain with good accuracy but in this case, the standard energy fluctuation method is quite effective. The use of the PDF complements traditional techniques utilizing energy fluctuations in Langevin simulations which are not suited to free energy and entropy calculations.

The QES nature of the DSHG theory allows not only the exact computation of $E_{0}$ at several temperatures, but 
also of $\partial E_{0} / \partial \beta$, using first order perturbation theory: $\partial E_{0} /\left.\partial \beta\right|_{\beta=\beta_{0}}=\left(\Psi_{0}, \partial^{2} \Psi_{0} / \partial \phi^{2}\right)$ where $\beta_{0}$ is one of the special checkpoint temperatures. Thus the internal energy $U$ and the entropy $S$ can also be found exactly at these temperatures. Once again, these quantities can be used to validate numerical work over a broad range of temperatures.

\section{CONCLUSION}

In this paper we have analytically obtained the free energy of the kink-bearing DSHG theory in $1+1$ dimensions at several temperatures. To our knowledge, this is the first such analytical calculation at several temperatures. We have compared the analytical results against large scale Langevin simulations and found excellent agreement between the two. In view of this agreement, the study of PDF-based thermodynamics via Langevin simulations appears quite tractable. It is worth emphasizing that the calibration of Langevin simulations constitutes a fundamental problem in the numerical study of nonlinear dynamical systems. Numerical convergence of results is certainly an important test that does not require the knowledge of an exact solution. However, standard finite difference error analysis cannot be applied directly to Langevin simulations because of their stochastic nature. Moreover, the error and convergence analysis for nonlinear stochastic PDEs with spatio-temporal noise remains to be completely worked out. For this reason, a nontrivial test system for which analytic solutions are available at several temperatures provides a valuable tool for testing the Langevin code. In fact based on our experience in the DSHG case, it is likely that previous Langevinbased analyses have errors as high as 30\%. We believe that apart from the intrinsic physical interest of the the double-well DSHG model, this system has the potential of becoming a standard benchmark problem in numerical simulations of Langevin systems.

We have also analytically studied the static equations of motion of the DSHG system and found an interesting connection between the $\phi^{4}$-theory, DSHG theory and a DSG theory. As a result, the kink and lattice kink solutions as well as kink total energy can be directly written down for any one of them in terms of the other. This is interesting as the lattice kink solution of this DSG case, which was unknown so far, can be immediately written down. Further, because of the intimate connection between the DSHG and the DSG case, it is reasonable to expect that a DSG theory may also be a QES system. We have been able to obtain several eigenstates of the DSG case at various temperatures from this identification [32]. It is clearly of great interest to carry out high quality Langevin simulations of the DSG case, which is a periodic system, and compare the agreement between the analytical and the simulation results. We hope to report on this problem in the near future.

\section{ACKNOWLEDGMENT}

This work was supported by the U.S. Department of Energy at Los Alamos National Laboratory. AK thanks the Theoretical Division of Los Alamos National Laboratory for hospitality. SH acknowledges useful discussions with Grant Lythe. Numerical simulations were performed on the CM-5 and the Origin 2000 at the Advanced Computing Laboratory, Los Alamos National Laboratory, and on the T3E at the National Energy Research Scientific Computing Center, Lawrence Berkeley National Laboratory.

\section{APPENDIX A: QES SOLUTIONS}

We obtain two sets of exact solutions for the Schrödinger equation (16).

Set-I $(n=1)$ : Note that $\epsilon_{0}$, as it occurs in the free energy, is related to the ground state energy $E_{0}$ by $\epsilon_{0}=E_{0}-V_{\min }$. For $n=1, V_{\min }=0$ for $\zeta<1$ while $V_{\min }=2 \zeta-\zeta^{2}-1$ for $\zeta>1$. However, in either case, $\epsilon_{n}-\epsilon_{m}=E_{n}-E_{m}$.

(1) For $2 \beta^{2}=1$ :

$$
\Psi_{0}(\phi)=\exp \left[-\frac{\zeta}{2} \cosh 2 \phi\right], E_{0}=1+\zeta^{2} .
$$

(2) For $2 \beta^{2}=4$ :

$$
\begin{aligned}
& \Psi_{0}(\phi)=\cosh \phi \exp [-\zeta \cosh 2 \phi], E_{0}=\zeta^{2}-\zeta+\frac{3}{4} \\
& \Psi_{1}(\phi)=\sinh \phi \exp [-\zeta \cosh 2 \phi], E_{1}=\zeta^{2}+\zeta+\frac{3}{4}
\end{aligned}
$$

Note that $\epsilon_{1}-\epsilon_{0}=E_{1}-E_{0}=2 \zeta$.

(3) For $2 \beta^{2}=9$ :

$$
\begin{aligned}
\Psi_{0}(\phi)= & {\left[6 \zeta+\left(1+\sqrt{1+36 \zeta^{2}}\right) \cosh 2 \phi\right] } \\
& \times \exp \left[-\frac{3 \zeta}{2} \cosh 2 \phi\right], \\
E_{0}= & \zeta^{2}-\frac{2}{9} \sqrt{1+36 \zeta^{2}}+\frac{7}{9}, \\
\Psi_{1}(\phi)= & \sinh 2 \phi \exp \left[-\frac{3 \zeta}{2} \cosh 2 \phi\right], \\
E_{1}= & \zeta^{2}+\frac{5}{9}, \\
\Psi_{2}(\phi)= & {\left[6 \zeta-\left(\sqrt{1+36 \zeta^{2}}-1\right) \cosh 2 \phi\right] } \\
\times & \exp \left[-\frac{3 \zeta}{2} \cosh 2 \phi\right] \\
E_{2}= & \zeta^{2}+\frac{1}{9}\left(2 \sqrt{1+36 \zeta^{2}}+7\right) .
\end{aligned}
$$


Note that $\epsilon_{1}-\epsilon_{0}=\frac{2}{9}\left(\sqrt{1+36 \zeta^{2}}-1\right)$ and $\epsilon_{2}-\epsilon_{0}=$ $\frac{4}{9} \sqrt{1+36 \zeta^{2}}$.

(4) For $2 \beta^{2}=16$ :

$$
\begin{aligned}
\Psi_{0}(\phi) & =\left[12 \zeta \cosh \phi+\left(2-4 \zeta+2 \sqrt{1-4 \zeta+16 \zeta^{2}}\right)\right. \\
& \times \cosh 3 \phi] \exp [-2 \zeta \cosh 2 \phi], \\
E_{0} & =\frac{1}{16}\left(16 \zeta^{2}-4 \sqrt{1-4 \zeta+16 \zeta^{2}}-8 \zeta+11\right) \\
\Psi_{1}(\phi) & =\left[12 \zeta \sinh \phi+\left(2+4 \zeta+2 \sqrt{1+4 \zeta+16 \zeta^{2}}\right)\right. \\
& \times \sinh 3 \phi] \exp [-2 \zeta \cosh 2 \phi] \\
E_{1} & =\frac{1}{16}\left(16 \zeta^{2}+8 \zeta-4 \sqrt{1+4 \zeta+16 \zeta^{2}}+11\right), \\
\Psi_{2}(\phi) & =\left[12 \zeta \cosh \phi+\left(2-4 \zeta-2 \sqrt{1-4 \zeta+16 \zeta^{2}}\right)\right. \\
& \times \cosh 3 \phi] \exp [-2 \zeta \cosh 2 \phi], \\
E_{2} & =\frac{1}{16}\left(16 \zeta^{2}-8 \zeta+4 \sqrt{1-4 \zeta+16 \zeta^{2}}+11\right) .
\end{aligned}
$$

Note that $\epsilon_{1}-\epsilon_{0}=\zeta+\frac{1}{4} \sqrt{1-4 \zeta+16 \zeta^{2}}-$ $\frac{1}{4} \sqrt{1+4 \zeta+16 \zeta^{2}}$ and $\epsilon_{2}-\epsilon_{0}=\frac{1}{2} \sqrt{1-4 \zeta+16 \zeta^{2}}$.

Set-II $(n=2)$ : In this case $V_{\min }$ for $\zeta>2$ is $4 \zeta-\zeta^{2}-4$ while $V_{\min }=0$ in case $\zeta<2$.

(1) For $2 \beta^{2}=1$ :

$$
\begin{aligned}
& \Psi_{0}(\phi)=\cosh \phi \exp \left[-\frac{\zeta}{2} \cosh 2 \phi\right], \quad E_{0}=\zeta^{2}-2 \zeta+3, \\
& \Psi_{1}(\phi)=\sinh \phi \exp \left[-\frac{\zeta}{2} \cosh 2 \phi\right], \quad E_{1}=\zeta^{2}+2 \zeta+3 .
\end{aligned}
$$

Note that $\epsilon_{1}-\epsilon_{0}=4 \zeta$.

(2) For $2 \beta^{2}=4$ :

$$
\begin{aligned}
\Psi_{0}(\phi) & =\left[3 \zeta \cosh \phi+\left(1-\zeta+\sqrt{1-2 \zeta+4 \zeta^{2}}\right) \cosh 3 \zeta\right] \\
& \times \exp [-\zeta \cosh 2 \phi], \\
E_{0} & =\frac{1}{4}\left(4 \zeta^{2}-4 \sqrt{1-2 \zeta+4 \zeta^{2}}-4 \zeta+11\right), \\
\Psi_{1}(\phi) & =\left[3 \zeta \sinh \phi+\left(1+\zeta+\sqrt{1+2 \zeta+4 \zeta^{2}}\right) \sinh 3 \phi\right] \\
& \times \exp [-\zeta \cosh 2 \phi], \\
E_{1} & =\frac{1}{4}\left(4 \zeta^{2}+4 \zeta-4 \sqrt{1+2 \zeta+4 \zeta^{2}}+11\right), \\
\Psi_{2}(\phi) & =\left[3 \zeta \cosh \phi+\left(1-\zeta-\sqrt{1-2 \zeta+4 \zeta^{2}}\right) \cosh 3 \phi\right] \\
& \times \exp [-\zeta \cosh 2 \phi], \\
E_{2} & =\frac{1}{4}\left(4 \zeta^{2}-4 \zeta+4 \sqrt{1-2 \zeta+4 \zeta^{2}}+11\right) .
\end{aligned}
$$

Note that $\epsilon_{1}-\epsilon_{0}=2 \zeta+\sqrt{1-2 \zeta+4 \zeta^{2}}-\sqrt{1+2 \zeta+4 \zeta^{2}}$ while $\epsilon_{2}-\epsilon_{0}=2 \sqrt{1-2 \zeta+4 \zeta^{2}}$.
(3) For $2 \beta^{2}=1 / 4$ :

$$
\Psi_{0}(\phi)=\exp \left[-\frac{\zeta}{4} \cosh 2 \phi\right], \quad E_{0}=\zeta^{2}+4 .
$$

(4) For $2 \beta^{2}=9 / 4$ :

$$
\begin{aligned}
\Psi_{0}(\phi) & =\left[3 \zeta+\left(1+\sqrt{1+9 \zeta^{2}}\right) \cosh 2 \phi\right] \\
& \times \exp \left[-\frac{3 \zeta}{4} \cosh 2 \phi\right] \\
E_{0} & =\zeta^{2}-\frac{8}{9} \sqrt{1+9 \zeta^{2}}+\frac{28}{9}, \\
\Psi_{1}(\phi) & =\sinh 2 \phi \exp \left[-\frac{3 \zeta}{4} \cosh 2 \phi\right], \\
E_{1} & =\zeta^{2}+\frac{20}{9} \\
\Psi_{2}(\phi) & =\left[3 \zeta-\left(\sqrt{1+9 \zeta^{2}}-1\right) \cosh 2 \phi\right] \\
& \times \exp \left[-\frac{3 \zeta}{4} \cosh 2 \phi\right] \\
E_{2} & =\zeta^{2}+\frac{4}{9}\left(2 \sqrt{1+9 \zeta^{2}}+7\right) .
\end{aligned}
$$

Note that $\epsilon_{1}-\epsilon_{0}=\frac{8}{9}\left[\sqrt{1+9 \zeta^{2}}-1\right]$ and $\epsilon_{2}-\epsilon_{0}=$ $\frac{16}{9} \sqrt{1+9 \zeta^{2}}$.

We now show that the first five and six levels of the DSHG potential as given by Eq. (2) can be written down exactly in case $n=5$ and $n=6$ respectively (at $2 \beta^{2}=$ $1)$. Using the trivial scaling, one can then obtain exact solutions for say $n=2$ but at $2 \beta^{2}=25 / 4$ and $2 \beta^{2}=9$. For example, for $n=5, \hat{E}_{0,2,4}$ are solutions of the cubic equation

$$
\hat{E}(\hat{E}+4)(\hat{E}+16)-64 \zeta^{2} \hat{E}-768 \zeta^{2}=0,
$$

and the corresponding eigenfunctions are given by

$$
\psi(\phi)=v(\phi) \exp \left[-\frac{\zeta}{2} \cosh 2 \phi\right]
$$

where

$$
\begin{aligned}
v_{0,2,4} & =6 \zeta-\hat{E} \cosh 2 \phi+\frac{2 \zeta \hat{E}}{\hat{E}+16} \cosh 4 \phi, \\
\hat{E}_{1,3} & =-10 \mp 2 \sqrt{4 \zeta^{2}+9} \\
v_{1,3} & =4 \zeta \sinh 2 \phi+\left(3 \pm \sqrt{4 \zeta^{2}+9}\right) \sinh 4 \phi .
\end{aligned}
$$

Note that here $\hat{E}=E-\zeta^{2}-25$.

For $\mathrm{n}=6, \hat{E}_{0,2,4}$ are solutions of the cubic equation

$$
\begin{aligned}
& (\hat{E}+9)(\hat{E}+25)(\hat{E}+6 \zeta+1)-52 \zeta^{2} \hat{E} \\
& -820 \zeta^{2}-120 \zeta^{3}=0
\end{aligned}
$$

and 


$$
\begin{aligned}
v_{0,2,4}= & 8 \zeta \cosh \phi-(\hat{E}+6 \zeta+1) \cosh 3 \phi \\
& +\frac{2 \zeta(\hat{E}+6 \zeta+1)}{\hat{E}+25} \cosh 5 \phi .
\end{aligned}
$$

Note that $\hat{E}_{1,3,5}$ are solutions of the same cubic equation with $\zeta$ replaced by $-\zeta$, and

$$
\begin{aligned}
v_{1,3,5}= & 8 \zeta \sinh \phi-(\hat{E}-6 \zeta+1) \sinh 3 \phi \\
& +\frac{2 \zeta(\hat{E}-6 \zeta+1)}{\hat{E}+25} \sinh 5 \phi .
\end{aligned}
$$

Here $\hat{E}=E-\zeta^{2}-36$.

[1] D. J. Scalapino, M. Sears, and R. A. Ferrell, Phys. Rev. B 6, 3409 (1972).

[2] J. A. Krumhansl and J. R. Schrieffer, Phys. Rev. B 11, 3535 (1975).

[3] A. G. Ushveridze, Quasi-exactly Solvable Models in Quantum Mechanics (IOPP, Bristol 1994); F. Cooper, A. Khare, and U. P. Sukhatme, Phys. Rep. 251, 267 (1995).

[4] A. Saxena and S. Habib, Physica D 107, 338 (1997).

[5] A. Khare, S. Habib, and A. Saxena, Phys. Rev. Lett. 79, 3797 (1997).

[6] A. D. Bruce, Adv. Phys. 29, 111 (1980).

[7] A. D. Bruce, T. Schneider, and E. Stoll, Phys. Rev. Lett. 43, 1284 (1979); A. D. Bruce, in Nonlinear Phenomena at Phase Transitions and Instabilities, edited by T. Riste (Plenum, New York, 1981); K. Binder, Z. Phys. B 43, 119 (1981).

[8] S. Habib and G. D. Lythe, (in preparation).

[9] M. Razavy, Phys. Lett. A72, 89 (1979); Am. J. Phys. 48, 285 (1980).

[10] S. N. Behera and A. Khare, J. Physique C6, 314 (1981).

[11] K. B. Joseph and B. V. Baby, J. Phys. A 16, 2685 (1983); B. Dey, J. Phys. A 19, 1735 (1986).

[12] P. T. Dinda, Phys. Rev. B 46, 12012 (1992).

[13] A. M. Dikandé and T. C. Kofané, Solid State Commun. 89, 283 (1994); ibid 559 (1994).

[14] H. Konwent, Phys. Lett. A 118, 467 (1986); H. Konwent, P. Machnikowski, and A. Radosz, J. Phys. A 28, 3757 (1995).

[15] R. K. Bullough, P. J. Caudrey, and H. M. Gibbs in Solitons, eds. R. K. Bullough and P. J. Caudrey (Springer-Verlag, Berlin, 1980), Chapt. 3; M. J. Ablowitz, M. D. Kruskal, and J. F. Ladik, SIAM J. Appl. Math. 36, 428 (1979).

[16] E. L. Ince, Proc. R. Soc. (Edinburgh) 45, 102 (1925).

[17] M. J. Ablowitz, D. J. Kaup, A. C. Newell, and H. Segur, Stud. Appl. Math. 53, 249 (1974); H. P. Mckean, Comm. Pure Appl. Math. 34, 197 (1981).

[18] S. E. Trullinger and R. M. DeLeonardis, Phys. Rev. B 20, 2225 (1979).

[19] J. F. Currie J. A. Krumhansl, A. R. Bishop, and S. E. Trullinger, Phys. Rev. B 22, 477 (1980).

[20] M. F. Manning, J. Chem. Phys. 3, 136 (1935).
[21] G. A. Baker, Jr. and A. R. Bishop, J. Phys. A 15, L201 (1982); G. A. Baker, Jr., A. R. Bishop, K. Fesser, P. D. Beale, and J. A. Krumhansl, Phys. Rev. B 26, 2596 (1982); J. H. Chen, M. E. Fisher, and B. G. Nickel, Phys. Rev. Lett. 48, 630 (1982); P. D. Beale, Phys. Rev. B 27, 5804 (1983).

[22] E. Matsushita and T. Matsubara, Prog. Theor. Phys. 67, 1 (1982); ibid 71, 235 (1984); E. S. Kryachko, Chem. Phys. 143, 359 (1990); O. Yanovitskii, G. Vlastou-Tsinganos, and N. Flytzanis, Phys. Rev. B 48, 12645 (1993).

[23] P. F. Byrd and M. D. Friedman, Handbook of Elliptic Integrals for Engineers and Scientists, 2nd ed. (Springer, Berlin, 1971).

[24] N. S. Manton, Nucl. Phys. B 150, 397 (1979).

[25] N. Gupta and B. Sutherland, Phys. Rev. A 14, 1790 (1976).

[26] B. Horovitz, Phys. Rev. B 35, 734 (1987); A. Saxena and W. Cao, Phys. Rev. B 38, 7664 (1988).

[27] F. J. Alexander, S. Habib, and A. Kovner, Phys. Rev. E 48, 4284 (1993).

[28] D. K. Campbell, M. Peyrard, and P. Sodano, Physica D 19, 165 (1986);

[29] R. M. Gundersen, Intern. J. Nonlin. Mech. 26, 389 (1991).

[30] A. Griner, W. Strittmatter, and J. Honerkamp, J. Stat. Phys. 51, 95 (1988).

[31] F. J. Alexander and S. Habib, Phys. Rev. Lett. 71, 955 (1993), and references therein.

[32] S. Habib, A. Khare, and A. Saxena (in preparation). 